\title{
A THEOREM ON RECURSIVELY ENUMERABLE CLASSES AND SPLINTERS ${ }^{1}$
}

\author{
PAUL R. YOUNG
}

Terminology. Except in a few cases which will be clear from their context, we reserve the word "class" to mean a set whose members are subsets of the set of nonnegative integers. We reserve the word "set" to mean a subset of $N$, the set of nonnegative integers. A class $\mathcal{H}$ of recursively enumerable (r.e.) sets is r.e. just in case there is an effective enumeration of indices for all members of $\mathfrak{H}$. A class $\mathfrak{g}$ of r.e. sets is called immune if it has no infinite r.e. subclass.

1. Introduction. The chief purpose of this paper is to give some examples which show how the theory of r.e. classes differs from the theory of r.e. sets.

In [2], Friedberg has shown that the class of all recursively enumerable sets is r.e. without repetition. In [3], Pour-El and Howard have provided a structural criterion which enables them to show that various r.e. classes are r.e. without repetition. It is clear that any such class must have proper infinite subclasses which are again r.e. without repetition. In [4], Pour-El and Putnam have given examples of r.e. classes which are not r.e. without repetition. They have pointed out that to obtain an infinite r.e. class having the property that every element is repeated infinitely of ten in every recursive enumeration of an infinite subclass of the class it would suffice to construct an infinite r.e. class which has no proper infinite r.e. subclass. In this paper we construct an infinite r.e. class, $\mathfrak{H}$, of r.e. sets which has no proper infinite r.e. subclass. Our proof uses the existence of maximal sets, [2], together with the technique which Friedberg has used to split nonrecursive r.e. sets into disjoint nonrecursive r.e. subsets, [2].

In [1, pp. 53-54], Dekker, Myhill, and Shoenfield construct two r.e. classes whose intersection is not r.e. Using techniques in [4], it is easy to obtain two r.e. classes whose intersection is immune. Here (with the aid of the referee) we show that for the r.e. class $\mathcal{H}$ mentioned above, there exists an $\alpha^{*} \notin \mathcal{H}$ such that for every $\alpha \in \mathfrak{F}$, $\mathfrak{H}_{\alpha}=(\mathfrak{K}-\{\alpha\}) \cup\left\{\alpha^{*}\right\}$ is r.e., but $\mathfrak{H} \cap \mathfrak{K}_{\alpha}(=\mathfrak{H}-\{\alpha\})$ is immune.

In [4], Pour-El and Putnam use their examples of r.e. classes to

Presented to the Society, December 29, 1965; received by the editors October 12, 1965.

1 Research and publication supported by a National Science Foundation Postdoctoral Fellowship at Stanford University and by NSF Grant 2754. 
obtain applications to r.e. sequences of formal theories. Our result together with their techniques yields similar applications: e.g., there exists a r.e. sequence $\delta$ of theories of identity (theories of Abelian groups, field theories, ring theories, integral domain theories, etc.) such that if $T \in \mathcal{S}$, then there is a theory $T^{*}$ of identity (Abelian groups, etc.) such that $T^{*} \notin \delta, \delta_{T}=(\delta-\{T\}) \cup\left\{T^{*}\right\}$ is r.e., but $S \cap S_{T}$ is immune. (In fact, just as with $\alpha^{*}$ and $\alpha$ in the preceding paragraph, $T^{*}$ may be chosen independently of $T$.)

We find it convenient to use splinters to visualize our construction of $\mathcal{H}$. Doing this enables us to incidentally prove the existence of a one-one total recursive function which has no recursive splinters. I.e., there is a one-one Turing machine which is defined for all inputs but which has the property that, no matter what initial input is taken, if the machine is successively given its outputs as new inputs, there is no decision procedure for determining whether or not an in teger belongs to the set of outputs.

2. Definitions. A component of a partial recursive function $f$ is a set of the form

$$
\left\{y \mid \exists i, j \geqq 0 \ni f^{i}(y)=f^{j}\left(x_{0}\right)\right\}
$$

for some integer $x_{0}$. We will use the notation

$$
\langle x, f\rangle \text { for }\left\{f^{i}(x) \mid i \in N \text { and } f^{i}(x) \text { is defined }\right\},
$$

where $N$ is the set of all nonnegative integers. If $f$ is a total recursive function, $\langle x, f\rangle$ is called an $f$-splinter, (Ullian). If $f$ is one-one and $f^{-1}(x)$ is not defined, we shall call $x$ the head of $\langle x, f\rangle$, and we shall say that $\langle x, f\rangle$ is headed.

3. Results. Our first lemma provides the motivation for attempting the construction given in the theorem which follows. The results mentioned in the introduction are then trivial consequences of the lemma and the theorem.

Lemma. Suppose $M$ is a maximal set and $f$ is a one-one total recursive function whose range is $M$. Suppose further that every component of $f$ intersects $M^{\prime}$ and that no splinter of $f$ is recursive. Then the class, $\mathfrak{H}$, of all headed $f$-splinters is an infinite r.e. class which has no proper infinite r.e. subclass.

Proof. Since each component intersects $M^{\prime}$ and $M$ is the range of $f$, the components of $f$ are exactly the headed $f$-splinters, and each of these has a head in $M^{\prime}$. Each headed $f$-splinter intersects $M^{\prime}$ exactly once. Since, given $x$, we can effectively enumerate the component of 
$f$ of which $x$ is a member, $\mathfrak{H}$ is an infinite r.e. class of r.e. sets. From the maximality of $M$, it follows that any infinite r.e. subclass of $\mathfrak{F C}$ would have to contain (indices for) all but finitely many members of F. But the sum set of these finitely many members of $\mathcal{H C}$ is certainly r.e., and if its complement were r.e., this sum set would be recursive. This in turn would imply that each of these finitely many members of अ would be recursive. This is a contradiction unless this finite subclass of $\mathfrak{H C}$ is empty, i.e. unless every infinite r.e. subclass of $\mathfrak{H C}$ is in fact $\mathfrak{H C}$ itself.

THEOREM. Let $R_{u}$ be any nonrecursive r.e. set. Then there exists a one-one total recursive function $f$ whose range is $R_{u}$ and which has the property that every component of $f$ is a nonrecursive splinter with a head in $R_{u}^{\prime}$.

REMARK. Clearly none of the splinters of $f$ can be recursive, for they all are contained in components of $f$ and hence differ from the headed $f$-splinters by finite sets.

Proof OF TheOREM. Following Friedberg's notation in Theorem 1 of [2], we shall denote the $e$ th r.e. set by $R_{e}$. We define the function $f$ by proceeding in steps, letting $B^{a}$ denote the set of integers placed in the set $B$ no later than Step $a$. We say that the number $e$ satisfies Condition $i$ at Step $a$ if $R_{e}^{a} \cap\langle i, f\rangle^{a}$ is not empty or if we have specified at an earlier step that $e$ satisfies Condition $i$ (because $i$ is found to be in $R_{u}$ ). We assume without loss of generality that $0 \in R_{u}^{\prime}$. For all sets $B$ in the construction, $B^{0}=\varnothing$.

Step $a(a \geqq 1)$. If there is no member of $R_{u}^{a}-R_{u}^{a-1}$, go on to Step $a+1$. Otherwise let $\{n\}=R_{u}^{a}-R_{u}^{a-1}$.

If there is no $e$ with $n \in R_{e}^{a}$ while $e$ fails to satisfy Condition $i$ for some $i \leqq \min (e, n-1)$, we let

$$
j=(\mu y)\left[f^{y}(0)\right. \text { is not yet defined], }
$$

and we define $f^{j}(0)=n$. This makes all $e$ satisfy Condition $n$, and we go on to Step $a+1$.

If there is an $e$ with $n \in R_{e}^{a}$ while $e$ fails to satisfy Condition $i$ for some $i \leqq \min (e, n-1)$, we let $e_{0}$ be the smallest such $e$ and $i_{0}$ be the smallest such $i$ (for $e_{0}$ ). We attack $e_{0}$ by defining $f^{j}\left(i_{0}\right)=n$, where

$$
j=(\mu y)\left[f^{y}\left(i_{0}\right) \text { is not yet defined }\right] \text {. }
$$

This makes $e_{0}$ satisfy Condition $i_{0}$, and it makes all $e$ satisfy Condition $n$. We go on to Step $a+1$.

This completes the description of the construction of $f$. Clearly each number $e$ gets attacked at most $e+1$ times. 
Now an immediate predecessor under $f$ of an integer $n$ is defined when and only when $n$ appears in $R_{u}^{a}-R_{u}^{a-1}$. Thus $f$ is a one-one partial recursive function whose range is $R_{u}$.

We now wish to show that for every integer $n, f^{-k}(n)$ is defined for at most finitely many integers $k$. Suppose that at the beginning of Step $a+1, n \in\langle m, f\rangle^{a}$ where $f^{-1}(m)$ has not yet been defined. Then in order to define $f^{-1}(m)$ during Step $a+1$, we must find $m$ in $R_{u}^{a+1}-R_{u}^{a}$ and define $f^{j}\left(i_{0}\right)=m$ for some $j$ and for some $i_{0}<m$. This puts $n$ in $\left\langle i_{0}, f\right\rangle^{a+1}$ where $f^{-1}\left(i_{0}\right)$ is not yet defined. Since $i_{0}<m$, this can happen only finitely of ten, so $f^{-k}(n)$ is defined for only finitely many values of $k$. Since $f^{-1}(n)$ is defined for all $n \in R_{u}$, it follows that every component of $f$ is a set of the form $\langle i, f\rangle$ for some $i \in R_{u}{ }^{\prime}$. If $f$ is a total recursive function, this means that every component of $f$ is a splinter with a head in $R_{u}^{\prime}$.

It remains to prove that $f$ is a total recursive function and that none of the sets $\langle i, f\rangle$ for $i \in R_{u}^{\prime}$ is recursive. It suffices to prove only the latter, for if $f(y)$ were not defined, recalling that $y \in\langle i, f\rangle$ for some $i \in R_{u}^{\prime}$, we would have that $\langle i, f\rangle$ would be finite and hence recursive. We may thus complete the proof by showing that for each $i \in R_{u}^{\prime}$, there is no r.e. set $R_{c}$ such that $\langle i, f\rangle \subseteq R_{c}^{\prime} \subseteq R_{u} \cup\{i\}$. To prove Proposition 2, which follows, it will be helpful to establish a slightly stronger relation. For each finite set $F$ and for each $i \in R_{u}^{\prime}$, there is no r.e. set $R_{c}$ such that

$$
\langle i, f\rangle \subseteq R_{c}^{\prime} \cup F \subseteq R_{u} \cup\{i\} \cup F .
$$

Suppose on the contrary that such an $i, F$, and $R_{c}$ exist. $R_{c}^{\prime} \cup F$ equals $\left(R_{c}-F\right)^{\prime}$; let $R_{d}=R_{c}-F$. Since $R_{d}$ has infinitely many indices, we may assume that $d>i$. Clearly $d$ never satisfies Condition $i$. Let $a_{0}$ be a step of the construction after which no $e \leqq d$ ever gets attacked. Now if any $n>i$ belongs to $R_{d}^{a}$ and is listed in $R_{u}^{a}-R_{u}^{a-1}$ for $a \geqq a_{0}$, then some $e \leqq d$ must be attacked, and this cannot occur.

Let $u_{0}, \cdots, u_{k}$ be those members of $R_{u}^{\prime}$ which are either less than or equal to $i$ or which belong to $F$. Let $u_{k+1}, \cdots, u_{m}$ be the members of $R_{u}^{\prime}$ which are listed in $R_{d}$ before Step $a_{0}$. Recall that $R_{u}^{\prime}-(\{i\} \cup F) \subseteq R_{d}$. Then

$$
y \in R_{u}^{\prime} \text { if and only if } y \in\left\{u_{0}, \cdots, u_{m}\right\} \text { or }
$$

$y>i$ and $y \notin R_{u}^{a}$ and $y$ is listed in $R_{d}^{a}-R_{d}^{a-1} \quad\left(a \geqq a_{0}\right)$.

This shows that $R_{u}^{\prime}$ would be r.e., and hence that $R_{u}$ would be recursive, a contradiction.

REMARK (ADDED MAY 1, 1966). Let $R_{u}$ be any nonrecursive r.e. 
set, let $f$ be constructed as in the preceding proof, and let $\mathfrak{F}$ be the class of all headed $f$-splinters. Then relation (I) implies that any proper r.e. subclass of $H$ differs infinitely from $\mathcal{H}$.

COROLlary 1. There is an infinite r.e. class of r.e. sets which has no proper infinite r.e. subclass.

COROLlARY 2. There is a one-one total recursive function $f$ which has no recursive splinters and for which all "splinters" of the partial recursive function $f^{-1}$ are finite. (I.e., all the sets $\left\langle i, f^{-1}\right\rangle$ for $i \in N$ are finite.)

COROLlary 3. There is an infinite r.e. class $\mathcal{H}$ of r.e. sets such that if $\alpha \in \mathfrak{H}$, then there exists an $\alpha^{*} \notin \mathfrak{H}$ with $\mathfrak{H}_{\alpha}=(\mathfrak{H}-\{\alpha\}) \cup\left\{\alpha^{*}\right\}$ r.e., but $\mathfrak{H C}_{\mathcal{H}} \cap \mathcal{H}_{\alpha}(=\mathfrak{H}-\{\alpha\})$ is immune.

Proof. Let $R_{u}$ be a maximal set and let $f$ and $\mathfrak{F}$ be as in the lemma and theorem. Let $\alpha \in \mathcal{F}$ and let $a_{0}$ be the head of $\alpha$. Take $\alpha^{*}=\left\langle f\left(a_{0}\right), f\right\rangle$. Since, given $x$, we can effectively enumerate all members of the component of $x$ but omitting $a_{0}, \mathfrak{H}_{\alpha}$ is r.e. The result now follows from the lemma and the theorem. (The referee has pointed out that, employing a technique of [4], we may instead take $\alpha^{*}$ to be $N$ and thus obtain the stronger result: For all $\alpha \in \mathcal{F}$, $\mathfrak{H}_{\alpha}=(\mathfrak{H}-\{\alpha\}) \cup\{N\}$ is r.e., but $\mathfrak{F C} \cap \mathfrak{H}_{\alpha}(=\mathfrak{H}-\{\alpha\})$ is immune. The same trick yields an improved version of Proposition 2.)

If $\mathcal{S}$ and $\mathcal{H}$ are r.e. classes such that $S \subseteq \mathcal{H}$ and $\mathfrak{H}-\mathcal{S}$ is infinite, then for any finite class $\mathfrak{F} \subseteq \mathfrak{F}-\delta, \delta \cup \mathcal{F}$ is r.e. Thus, to construct r.e. classes with only finitely many proper infinite r.e. subclasses, all of the infinite r.e. subclasses must differ from the given classes by only finitely many members.

Proposition 1. Let $n$ be any integer which can be written as a product of integers of the form $2^{m}$ or $2^{m}-1$. Then there exists a r.e. class of r.c. sets which has exactly $n$ distinct infinite r.e. subclasses.

Proof. Let $R_{u}$ be a maximal set and let $f$ and $\mathcal{H}$ be as in the lemma and theorem. Let $r_{0}, r_{1}, \cdots, r_{k}$ be any integers with $r_{0} \geqq 0$ and $r_{j} \geqq 1$ for $1 \leqq j \leqq k$. Let $\mathfrak{H}=\left\{\alpha_{0}, \alpha_{1}, \cdots, \alpha_{k}, \alpha_{k+1}, \cdots\right\}$, with $\alpha_{j}=\left\langle a_{j}, f\right\rangle$ for all $j$. Define

$$
\mathfrak{F}^{*}=\mathfrak{H} \cup\left\{\left\{f^{i}\left(a_{0}\right)\right\} \mid 1 \leqq i \leqq r_{0}\right\} \cup\left\{\left\langle f^{i}\left(a_{j}\right), f \supset\right| 1 \leqq j \leqq k ; 1 \leqq i \leqq r_{j}-1\right\} .
$$

$\mathfrak{H C}^{*}$ is clearly r.e., and one can prove that $\mathfrak{H}^{*}$ has $2^{r_{0}} \cdot\left(2^{r_{1}}-1\right) \ldots$ $\left(2^{r_{k}}-1\right)$ infinite r.e. subclasses. The crucial facts, which are verified as in the first part of the proof of the theorem, are these: every infinite r.e. subclass of $\mathfrak{F}^{*}$ includes $\left\langle a_{0}, f\right\rangle$ and may include any com- 
bination at all of $\left\{f\left(a_{0}\right)\right\}, \cdots,\left\{f^{r}\left(a_{0}\right)\right\}$, and for each $1 \leqq j \leqq k$ every infinite r.e. subclass of $\mathfrak{H}^{*}$ includes at least one of $\left\langle a_{j}, f\right\rangle, \cdots$, $\left\langle f^{r_{j}-1}\left(a_{j}\right), f\right\rangle$ but may contain any combination of these in which at least one is included.

We obtain from this proposition the existence of r.e. classes with exactly $1,2,3,4,6,7,8,9,12, \cdots$, infinite r.e. subclasses. Ad hoc arguments provide other values; e.g. if $R_{u}$ is maximal and if $\mathfrak{H}$ $=\left\{\alpha_{0}, \alpha_{1}, \alpha_{2}, \ldots\right\}$ is as in the theorem and lemma, then $\mathfrak{F}^{*}$ $=\mathscr{H} \cup\left\{\alpha_{0} \cup \alpha_{1}\right\}$ has exactly five infinite r.e. subclasses; it is easily seen how to combine this technique with the techniques used in the proof of the preceding proposition to obtain r.e. classes with 10, 15, $20,25, \cdots$ infinite r.e. subclasses, (but not, e.g., 55). We would like to know whether all positive integers are possible values for the number of infinite r.e. subclasses of r.e. classes. (Added May 1, 1966: This question has been answered affirmatively by John Florence, a student of A. H. Lachlan.)

Proposition 2. There exists a r.e. sequence $S$ of theories of identity (theories of Abelian groups, field theories, ring theories, integral domain theories, etc.) such that if $T_{k} \in \mathcal{S}$, then there exists a theory $T_{k}^{*}$ of identity (of Abelian groups, etc.) such that $T_{k}^{*} \notin S$ and $S_{k}=\left(S-\left\{T_{k}\right\}\right) \cup\left\{T_{k}^{*}\right\}$ is r.e., but $\mathrm{S} \cap \mathrm{S}_{k}\left(=\delta-\left\{T_{k}\right\}\right)$ is immune.

Proof. Let $R_{c}$ be the set of all prime numbers and let $R_{u}$ be a maximal set such that $R_{c}^{\prime} \subseteq R_{u}$. Let $\mathcal{F C}$ and $f$ be as in the theorem and lemma, and let $\mathfrak{F C}=\left\{\alpha_{0}, \alpha_{1}, \cdots, \alpha_{n}, \cdots\right\}$. Following [4], let $\Phi_{n}$ express the proposition that there are exactly $n$ elements in the domain. For theories of identity, the axioms of the theory $T_{k}$ are exactly those sentences $\rceil \Phi_{i}$ for which $i \in \alpha_{k}$. From the relation (I) in the proof of the theorem, we see that each $\alpha_{k}$ contains infinitely many primes. Let $p_{k}$ be some prime number belonging to $\alpha_{k}$. We define $\alpha_{k}^{*}$ to be $\alpha_{k}-\left\{p_{k}\right\}$, and we then take as axioms for the theory $T_{\boldsymbol{k}}^{*}$ exactly those sentences $\rceil \Phi_{i}$ for which $i \in \alpha_{k}{ }^{*}$.

For the various algebraic theories, we take as axioms for $T_{k}$ and $T_{k}^{*}$ not only the axioms of identity as given above but also the appropriate algebraic axioms. In each case $S=\left\{T_{k} \mid k \geqq 0\right\}$, and for each $T_{k} \in \mathcal{S}$ we take $S_{k}=\left(\mathcal{S}-\left\{T_{k}\right\}\right) \cup\left\{T_{k}^{*}\right\}$. The techniques which enable us to now verify the proposition are straightforward, and the reader may find them applied to obtain similar results in [4].

As a final remark, note that Theorem 13 of [4] is applicable to each of the sequences $\delta$ mentioned in the preceding proposition. Thus each of these sequences has an interesting superclass which is r.e. without repetitions. 


\section{REFERENCES}

1. J. C. E. Dekker and J. Myhill, Some theorems on classes of recursively enumerable sets, Trans. Amer. Math. Soc. 89 (1958), 25-59.

2. Richard Friedberg, Three theorems on recursive enumeration, J. Symbolic Logic 23 (1958), 309-316.

3. Marian Pour-El and William Howard, A structural criterion for recursive enumeration without repetition, Z. Math. Logik Grundlagen Math. 10 (1964), 105-114.

4. Marian Pour-El and Hilary Putnam, Recursively enumerable classes and their application to recursive sequences of formal theories, Arch. Math. Logik Grundlagenforsch. 8 (1965), 104-121.

Purdue University 